\title{
Clostridium leptum group bacteria abundance and diversity in the fecal microbiota of patients with inflammatory bowel disease: a case-control study in India
}

Jayakanthan Kabeerdoss, Vijayalakshmi Sankaran, Srinivasan Pugazhendhi and Balakrishnan S Ramakrishna*

\begin{abstract}
Background: Alterations in the fecal bacterial flora occur in inflammatory bowel disease (IBD). We examined the abundance and diversity of Clostridium leptum group, an important group of carbohydrate-fermenting bacteria, in the feces of patients with IBD and compared them with healthy controls.

Methods: Seventeen healthy controls (HC), 20 patients with Crohn's disease (CD) and 22 patients with ulcerative colitis (UC) participated in the study. DNA extracted from fecal samples was amplified by PCR targeting 165 rRNA gene sequences specific to C. leptum group. The PCR product was subjected to temporal temperature gradient electrophoresis (TTGE) and the number and position of individual bands were noted and diversity was estimated. The identity of bands at different positions was confirmed by cloning and sequencing. Real time quantitative PCR with Mesa Green, targeted at specific 16S rRNA gene sequences, was used to quantitate C. leptum group and its most prominent constituent, Faecalibacterium prausnitzii.
\end{abstract}

Results: Twenty five different operational taxonomic units (OTUs, equivalent to species) were identified constituting the $C$. leptum group in these participants. Their sequences were deposited in GenBank [accession numbers GQ465348 to GQ465370]. OTU number was significantly reduced in CD (7.7 \pm 3.7 , mean $\pm S D)$ and UC (9.0 \pm 3.0$)$ compared to HC (11.9 \pm 2.2$)(P=0.0005)$. The Simpson $D$ index of alpha diversity was not significantly different between the three groups. Total numbers of $C$. leptum group bacteria and F. prausnitzii were reduced in both $C D$ and $U C$ compared to $H C$ ( $P=0.0036$ and $P<0.0001$ respectively). Disease activity did not influence numbers of C. leptum or F. prausnitzii in patients with CD or UC.

Conclusion: C. leptum numbers and diversity were significantly reduced in both CD and UC suggesting that alterations noted were not specific to one disease. This could contribute to reduced short chain fatty acid production in IBD.

Keywords: Clostridial cluster IV, Dysbiosis, Crohn's disease, Ulcerative colitis, Short chain fatty acids

\footnotetext{
* Correspondence: rama@cmcvellore.ac.in

The Wellcome Trust Research Laboratory, Department of Gastrointestinal

Sciences, Christian Medical College, Vellore 632004, India
} 


\section{Background}

The Clostridium leptum group of bacteria is one of the dominant groups of fecal bacteria in adult humans, constituting $16-25 \%$ of the fecal microbiota [1,2]. This group, also called Clostridial cluster IV, includes Faecalibacterium prausnitzii and certain species of Eubacterium and Ruminococcus [3]. Members of this group synergize with other intestinal microbiota to ferment unabsorbed dietary carbohydrate, producing short chain fatty acids of which butyrate is the major energy source for the colonic epithelium and profoundly influences intestinal epithelial function. F. prausnitzii, the most abundant member of this group, is a major producer of butyrate through carbohydrate fermentation [4]. It has acquired special status because of its association with antiinflammatory effects in the gastrointestinal tract [5]. Any alteration in the composition of the gut microbiota that changes the microbial balance is likely to have repercussions on human health.

Inflammatory bowel diseases (IBD), comprising the two diseases ulcerative colitis (UC) and Crohn's disease (CD) are thought to result from disordered innate immune reactions to luminal microbiota in individuals with the appropriate genetic disposition [6]. A number of studies indicate that microbiota alterations, termed dysbiosis, are common in IBD [7-14]. These alterations include reduction of total microbial diversity, increased numbers of Enterobacteriaceae, increased abundance of Bacteroides-Prevotella, increase or decrease in bifidobacteria, and decrease in the numbers of Firmicutes (especially Clostridium coccoides-Eubacterium rectale and $F$. prausnitzii). Most of the changes described have been in patients with active CD. An earlier study suggested that $F$. prausnitzii numbers were decreased in active $C D$ but increased in active UC [8]. It is not known whether these changes are specific to $\mathrm{CD}$, or whether they reflect changes that are more universally present in patients with IBD. It is also not known whether these changes in the microbiota persist in quiescent IBD.

In the present study we investigated the species diversity of the C. leptum group of bacteria in the feces of healthy individuals and patients with IBD in remission using amplification of the $16 \mathrm{~S}$ rRNA gene followed by temperature gradient electrophoresis. We also quantitated C. leptum group and F. prausnitzii using real time PCR targeted at the $16 \mathrm{~S}$ rRNA gene sequences specific to these bacterial communities.

\section{Methods}

\section{Participants}

Seventeen healthy control (HC) volunteers, 20 patients with $\mathrm{CD}$ and 22 patients with UC participated in this study. Participants were identified in the IBD clinic of the department, informed about the purpose of the study, and provided written consent. CD and UC were diagnosed on the basis of Asian consensus criteria for diagnosis of these diseases [15]. Disease activity status was assessed by the Crohn's disease activity index and Truelove-Witts scoring system respectively [16,17]. Controls were identified from apparently healthy individuals visiting the general medical clinics of the hospital for routine health checkup. Participants who had taken antibiotics within the last three months were excluded. Fresh samples of feces were collected and transported within three hours to the laboratory, where they were stored in aliquots at $-20^{\circ} \mathrm{C}$ for processing in batches. The protocol was approved by the institutional ethics committee of the Christian Medical College, Vellore.

\section{DNA extraction and polymerase chain reaction amplification}

Total DNA was extracted from $250 \mathrm{mg}$ (wet weight) of fecal sample using the QIAamp DNA stool mini kit (Qiagen, Hilden, Germany) as per manufacturer's instructions. The DNA was eluted in a final volume of $200 \mu \mathrm{L}$ and stored at $-20^{\circ} \mathrm{C}$. The concentration and integrity of the nucleic acids were determined by electrophoresis on 1\% agarose gel containing ethidium bromide. C. leptum group DNA sequences were amplified using primers that targeted sequences in the $16 \mathrm{~S}$ rRNA gene specific to that group. The primers that were used were sg-Clept-F (Forward 5'-GCACAAGCAGTG GAGT-3') and Clept-GC-R3 (Reverse with GC Clamp 5'-CTTCCTCCGTTTTGTCA-3') [18]. Polymerase chain reaction (PCR) was performed in a reaction volume of $20 \mu \mathrm{l}$ containing 1X PCR buffer, $2.5 \mathrm{mM} \mathrm{MgCl}$, $200 \mathrm{mM}$ each dNTP, $250 \mathrm{nmol}$ of both forward and reverse primers, $2.5 \mathrm{U}$ of Jumpstart Taq polymerase (Sigma Aldrich Co, St. Louis, MO, USA), and approximately 10 ng of DNA template. Samples were amplified in a MJ Mini thermal cycler (Bio-Rad, Hercules, CA, USA) using the following program: $95^{\circ} \mathrm{C}$ for 1 minutes, 30 cycles of $95^{\circ} \mathrm{C}$ for 30 seconds, $56^{\circ} \mathrm{C}$ for 30 seconds, $72^{\circ} \mathrm{C}$ for 30 seconds, and finally $72^{\circ} \mathrm{C}$ for 10 minutes. PCR products were analyzed by electrophoresis on $2 \%$ agarose gel containing ethidium bromide to check their size (239 bp) and estimate their concentration.

\section{Temporal temperature gradient electrophoresis}

We subjected the PCR product to temporal temperature gradient electrophoresis (TTGE) for sequence-specific separation. Electrophoresis was performed in $1 \mathrm{~mm}$ thick, $16 \times 16 \mathrm{~cm}$ polyacrylamide gel $(7 \% \mathrm{wt} / \mathrm{vol}$ acrylamide/Bis, $7 \mathrm{M}$ urea, 1.25X TAE, and, respectively, $55 \mathrm{ml}$ and $550 \mu \mathrm{l}$ of TEMED and 10\% ammonium persulfate) using 25 litres of 1.25X TAE as the electrophoresis buffer in a TTGE detection system (CBS Scientific Co, San Diego, CA, USA). The TTGE conditions included electrophoresis at $50 \mathrm{~mA}$ 
for $12 \mathrm{~h}$ at an initial temperature of $66^{\circ} \mathrm{C}$ and a ramp rate of $0.3^{\circ} \mathrm{C} / \mathrm{h}$. To improve resolution, the voltage was set at $20 \mathrm{~mA}$ for $15 \mathrm{~min}$ at the beginning of each run. Each well was loaded with 100 to 200 ng of amplified DNA plus an equal volume of $2 \mathrm{X}$ gel loading dye $(0.05 \%$ bromophenol blue, $0.05 \%$ xylene cyanol, and $70 \%$ glycerol). Gels were stained in the dark by immersion for $15 \mathrm{~min}$ in a solution of ethidium bromide stain and were read using the Chemicapture software in a Chemi-Smart imaging system (Vilber Lourmat, Marne-la-Vallee, France). Each sample analyzed in this study was run in duplicate and consensus data compiled. In order to determine variations between PCR and TTGE, DNA samples from a subset of subjects were amplified in duplicate by PCR and TTGE was run again on another day. These samples were compared to each other by TTGE on the same gel, and PCR amplicons were run again on a different day and gel to determine gel-to-gel variation. Composite data sets for groupspecific TTGE profiles were generated and numerical band matching character tables produced. The BioDiversity Pro statistical package (version 2; Scottish Association for Marine Science, http://www.sams.ac.uk) was used for principal component analysis. The SDR software (version 4.1.2, Pisces Conservation Ltd., www.pisces-conservation.com) was used for diversity analysis.

\section{Cloning of the PCR products and clone library analysis}

Cloning PCR was performed using the same primers but without the GC clamp in the reverse primer. The purified PCR products were cloned into E. coli DH5 $\alpha$ using the $\mathrm{pCR}^{\mathrm{TM}}$ 2.1-TOPO ${ }^{\mathrm{R}}$ vector (TOPO TA cloning kit, Invitrogen, Carlsbad, CA, USA). Forty five recombinant clones were randomly selected for analysis. The plasmids were purified (Purelink Plasmid Miniprep Kit, Invitrogen, Carlsbad, CA, USA) and were used as templates for amplification of the $239 \mathrm{bp}$ C. leptum group-specific 16S rRNA gene fragment with the above mentioned primers without GC clamp for reverse primers. The amplicons were subjected to TTGE analysis. If two clones migrated to the same distance, only one clone was selected for sequencing. All clones which migrated to different distances were selected for sequencing. A total of 23 clones were sequenced using M13 forward primer (Bioserve Biotechnologies, Hyderabad, India). Clones which were sequenced were run on the TTGE analysis along with one sample (individual A) to identify the phylogeny of individual bands on TTGE.

\section{Sequence analysis}

Sequences from our clones and sequences retrieved from the GenBank database were taken to a length of 239 bp corresponding to the C. leptum amplicon length. Sequences obtained in our study were aligned with the sequences in the Ribosomal Database Project (release 9) database to determine their closest relatives. Sequences were aligned separately using ClustalX 2.0.9 and analyzed using PHYLIP version 3.68 after 1000 bootstrapping operations. The distance matrices on these datasets were created using DNADIST (PHYLIP version 3.68) and the sequences were subsequently clustered using NEIGHBOUR (PHYLIP version 3.68). The tree files from NEIGHBOUR were applied with CONSENSE (PHYLIP version 3.68) and the consensus tree was viewed using PHYLODRAW.

\section{Real time PCR assays for C. leptum group and Faecalibacterium prausnitzii}

Real-time quantitative (q) PCR was performed using a Chromo4 four color real time PCR detection system with Opticon Monitor 3 software (Bio-Rad, Hercules, USA). Amplification and detection were carried out in 96-well plates with Mesa Green qPCR Master Mix (Eurogentec SA, Liege, Belgium). Primers were directed at $16 \mathrm{~S}$ rRNA sequences specific to $C$. leptum and F. prausnitzii. The primers used to quantify $C$. leptum were the same as those used for TTGE but without the GC clamp in the reverse primer. The primers used to quantify $F$. prausnitzii were as follows: Forward primer $5^{\prime}$-GGAGGATTGACCCCTT CAGT- $3^{\prime}$ and reverse primer $5^{\prime}$-CTGGTCCCGAAGAAA CACAT-3' [19]. Primers were synthesized and purchased from Sigma Aldrich Chemicals (Bangalore, India). Each reaction was done in duplicate in a final volume of $20 \mu \mathrm{L}$. Amplifications were performed as follows: $95^{\circ} \mathrm{C}$ for 5 minutes, to denature DNA and followed by 40 cycles of $94^{\circ} \mathrm{C}$ for 10 seconds, $58^{\circ} \mathrm{C}$ for 30 seconds and $72^{\circ} \mathrm{C}$ for 30 seconds. The final extension step at $72^{\circ} \mathrm{C}$ for $10 \mathrm{~min}$ was followed by a melting curve analysis which was performed by increasing the temperature from 40 to $95^{\circ} \mathrm{C}$, with a raise in temperature by $1^{\circ} \mathrm{C}$ every $10 \mathrm{sec}$ with a plate read step to read the fluorescent signal. DNA copy was expressed as copy number per gram of feces and samples were run in real time PCR along with plasmid standards.

\section{Statistical analysis}

Normally distributed data was compared using ANOVA with post-hoc Bonferroni tests, while data that was not distributed normally was compared using the Kruskal-Wallis test with post-hoc Dunn tests.

\section{Results}

Twenty CD patients (6 female) with mean (SD) age of 31.2 (14.1) years were recruited. They had disease duration ranging from 6 months to three years. Eleven of the patients had a CDAI score of 150 or more, and were categorized as having active CD. Twenty two UC patients ( 9 female) with mean (SD) age 38.4 (11.3) years were recruited. They had disease duration ranging from six months to eight years. Nine of the UC patients had a UCAI score of 3 or more, and were categorized as having active UC. None of the patients was on treatment with 
antibiotics. All the IBD patients received treatment with aminosalicylates. Seven of the CD patients and six of the $\mathrm{UC}$ patients also received azathioprine. Four of the CD patients had undergone surgery earlier. None of the patients had colonic lavage or colonoscopy within the month prior to stool collection. Seventeen HC (8 female) with mean (SD) age 31.1 (13.8) years were recruited.

\section{Clone library analysis of $C$. leptum cluster and identification of TTGE bands}

A total of 45 clones were obtained which were amplified and run on TTGE. Only clones which differed in migrating position were selected for sequencing. Thus 25 clones were sequenced. The analysis showed that our cloned sequences had high similarity with known sequences available in GenBank. Most of the clones that we isolated belonged to genus Faecalibacterium (65\%), Subdoligranulum (8\%) and Ruminococcus (4\%). The recombinant clones in the library were amplified using primers sg-Clept-F and sg-Clept-GCR3, and the amplified products were subjected to TTGE in order to generate the identity of each of the TTGE bands.

\section{Nucleotide sequence accession numbers}

The sequences generated in this study were deposited in the GenBank database under accession numbers [GQ465348 to GQ465370].

\section{TTGE profiles for C. leptum group}

The PCR amplicons were run on TTGE (Figure 1), the image documented and a binary data matrix created by the presence or absence of bands at a specific location. Each band was considered as an operational taxonomic unit (OTU) equivalent to a species. Totally we observed 25 species for the C. leptum group by TTGE.

\section{Multivariate analysis of the TTGE profiles}

Principal component analysis (PCA) of the TTGE profiles obtained from the healthy subjects and IBD patients demonstrated that the bacterial community profiles were dissimilar by the first and the second principal component ( $x$ and $y$ axes on the PCA plot, respectively) (Figure 2). $\mathrm{HC}$ were predominantly grouped to the right side of PCA1 whereas CD and UC were more likely to cluster on the left side in PCA1, suggesting that the microbial communities of the healthy individuals shared some characteristics that differentiated them from IBD patients. $\mathrm{CD}$ and $\mathrm{UC}$ appeared to cluster together in the plot.

\section{Cluster analysis of the TTGE data}

The TTGE binary data, that is the presence or absence of bands, was analyzed by cluster analysis using Bray's cluster index (Figure 3). In this analysis all sample data were analyzed together, including $\mathrm{HC}, \mathrm{CD}$ and $\mathrm{UC}$. Compared to the healthy controls, most of the $\mathrm{CD}$ and UC patients showed higher dissimilarity in the dendrogram, which again confirmed the observation of PCA. At cut-off $70 \%$ below that similarity, controls and IBD groups were clearly differentiated.

\section{Diversity indices based on TTGE profile}

The number of $C$. leptum group species (Figure 4) was significantly less in CD and UC compared to HC. HC had a mean (SD) of 11.9 (2.2) OTUs in the C. leptum group compared to 7.7 (3.7) in CD patients and 9.0 (3.0) in UC patients $(\mathrm{P}<0.001 \mathrm{HC}$ vs. $\mathrm{CD} ; \mathrm{P}<0.05 \mathrm{HC}$ vs. UC; P-NS CD vs. UC). The Simpson's D index of alpha diversity was 19.65 for $\mathrm{HC}$ compared to 17.56 for $\mathrm{CD}$ and 17.51 for UC. The difference between $\mathrm{HC}$ and CD and UC was not statistically significant.

\section{Quantitation of C. leptum group and Faecalibacterium prausnitzii in feces}

The count of Clostridium leptum group bacteria in feces was significantly reduced in $\mathrm{CD}$ and $\mathrm{UC}$ compared to healthy controls ( $\mathrm{HC}$ vs. $\mathrm{CD} \mathrm{P}<0.01 ; \mathrm{HC}$ vs. $\mathrm{UC} \mathrm{P}<0.05)$ (Figure 5A). Faecalibacterium prausnitzii counts were

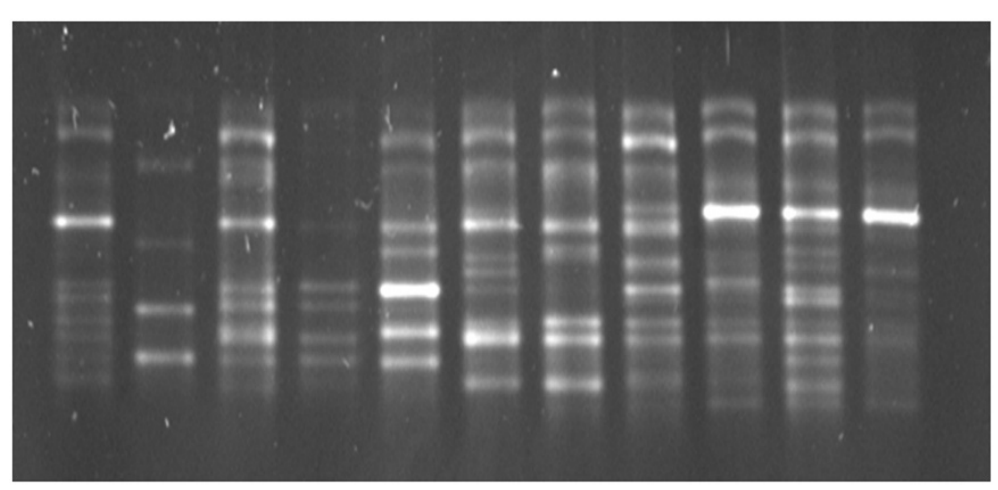

Figure 1 TTGE gel showing bands migrating at different positions. Each lane depicts fecal DNA amplified using the Clept primers from an individual participant. Details of the TTGE conditions are provided in the text. 


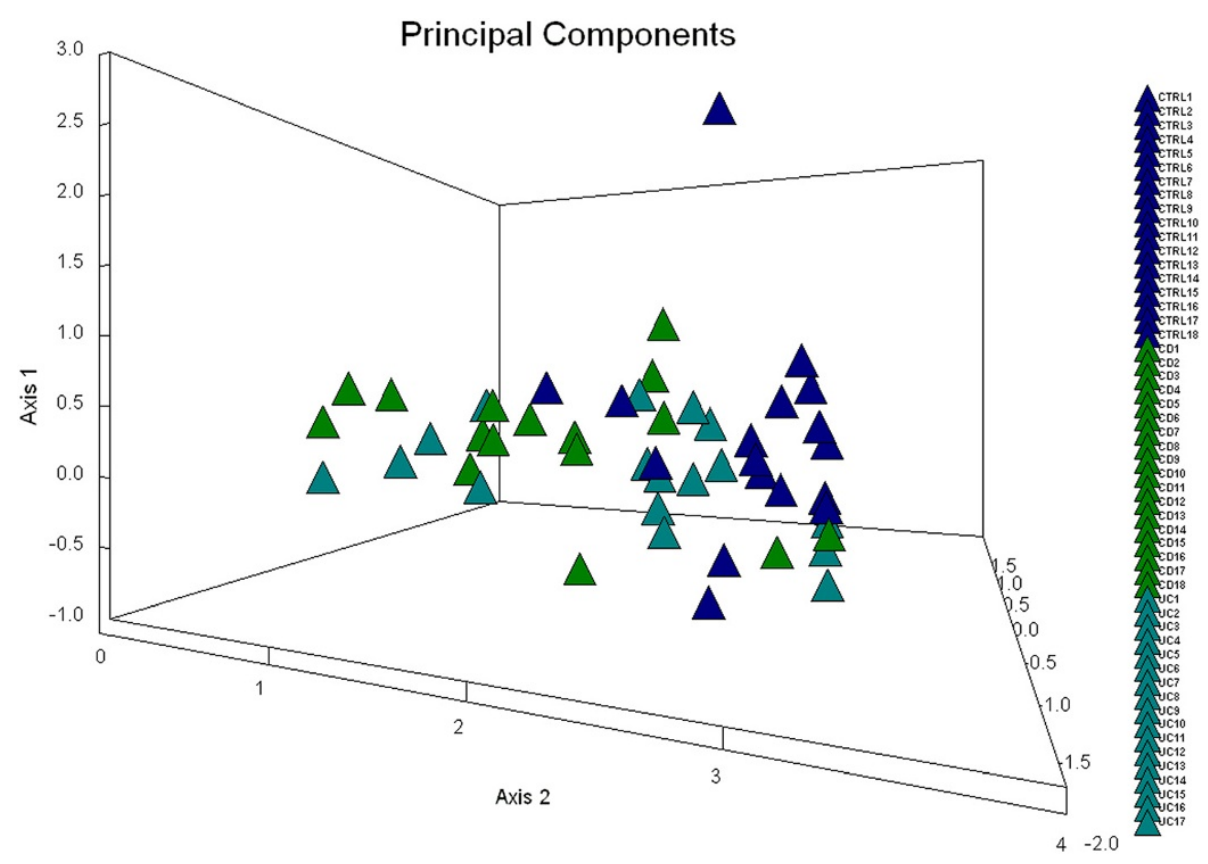

Figure 2 Principal components analysis of fecal C. leptum group bacterial communities. C. leptum species dominantly represented in healthy controls ( $\mathrm{HC}$, blue) tend to cluster to the right side of the plot, while those species dominant in Crohn's disease (CD, green) and ulcerative colitis (UC, turqoise) patients tend to cluster to the left of the plot.

also significantly reduced in $\mathrm{CD}$ and UC patients compared to $\mathrm{HC}$ ( $\mathrm{HC}$ vs. $\mathrm{CD} \mathrm{P}<0.001$; healthy vs. $\mathrm{UC} \mathrm{P}<0.001)$ (Figure 5B).

\section{Microbiota composition: comparison of active IBD with disease remission}

Comparison of $C$. leptum group and F. prausnitzii quantitation between IBD patients with active disease $(\mathrm{CDAI} \geq 150$ or $\mathrm{UCAI} \geq 3$ ) and those in clinical remission $(\mathrm{CDAI}<150$ or $\mathrm{UCAI}<3$ ) did not show any quantitative differences between the two groups (Figure 6). There was no correlation of microbial community abundance with duration of diarrhea.

\section{Discussion}

This study, using TTGE to study diversity of C. leptum group in IBD patients, provides important information regarding the colonic microbiota in IBD that will augment the existing knowledge in this area. The study shows that there was a reduction of absolute number as well as species number per individual of the C. leptum group in both UC and CD. The reduction in numbers was explained to a large extent by the reduction in $F$. prausnitzii, and this alteration was present in both quiescent and active IBD. C. leptum group bacteria from IBD patients clustered separately from bacteria present in the healthy controls. Lastly the study provides, for the first time, a catalog of the C. leptum group of bacteria in the feces of healthy adult humans in India. The present study focused solely on the C. leptum group and $F$. prausnitzii and did not attempt to determine changes in other microbial communities in IBD.

The utilization of culture-independent methods of analysis has significantly enhanced our ability to assess the contribution made by the gut microbiota to human health. In this study, we amplified portions of the $16 \mathrm{~S}$ rRNA gene that are specific for the C. leptum group of bacteria and used TTGE to identify the numbers of species present in in each individual. TTGE is a very sensitive technique to detect single nucleotide changes in DNA. The primer set used is able to detect species of the C. leptum group including C. leptum, Eubacterium plautii, Clostridium viride, and Ruminococcus albus strains which are present in small proportions in human fecal samples [19]. TTGE revealed multiple bands, which were cloned and sequenced to reveal at least 25 different species or OTU belonging to this group. The clone library that we generated, as well as the real time PCR data, showed that $F$. prausnitzii was the major constituent (approximately 65\%) of this bacterial group. The presence of Subdoligranulum variabile as a part of this bacterial group in our participants is consistent with other reports [20].

Healthy controls in the present study had a mean of 12 OTUs of the C. leptum group in feces, and this number was significantly reduced in CD and UC patients. However, using the Simpson D index, we did not find any alteration in alpha diversity between $\mathrm{HC}, \mathrm{UC}$ and CD. 


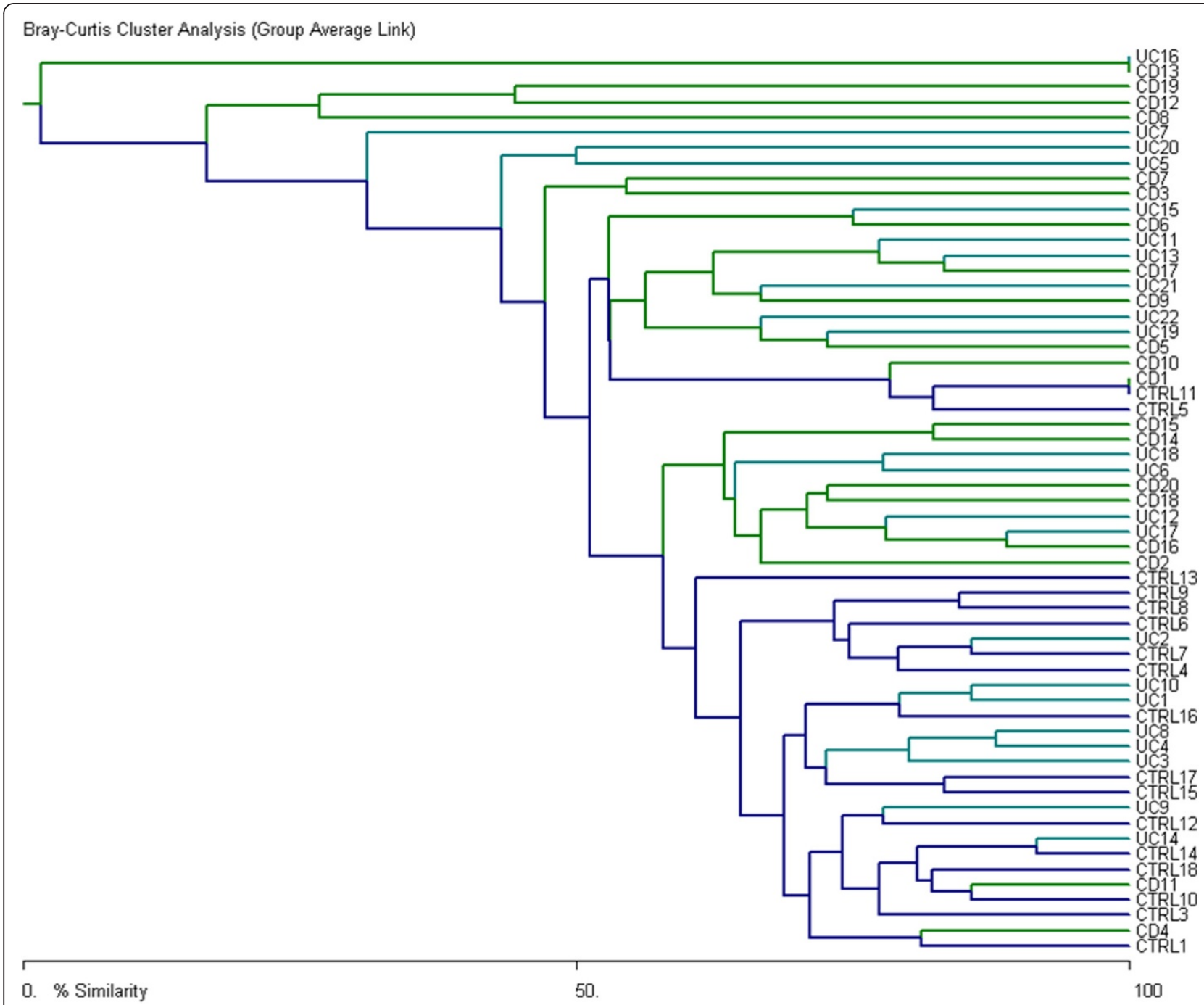

Figure 3 Bray Curtis cluster analysis of fecal C. leptum group communities. Bacteria from Crohn's disease (CD) and ulcerative colitis (UC) patients appeared to have higher degrees of dissimilarity to each other than bacteria from healthy controls (HC).

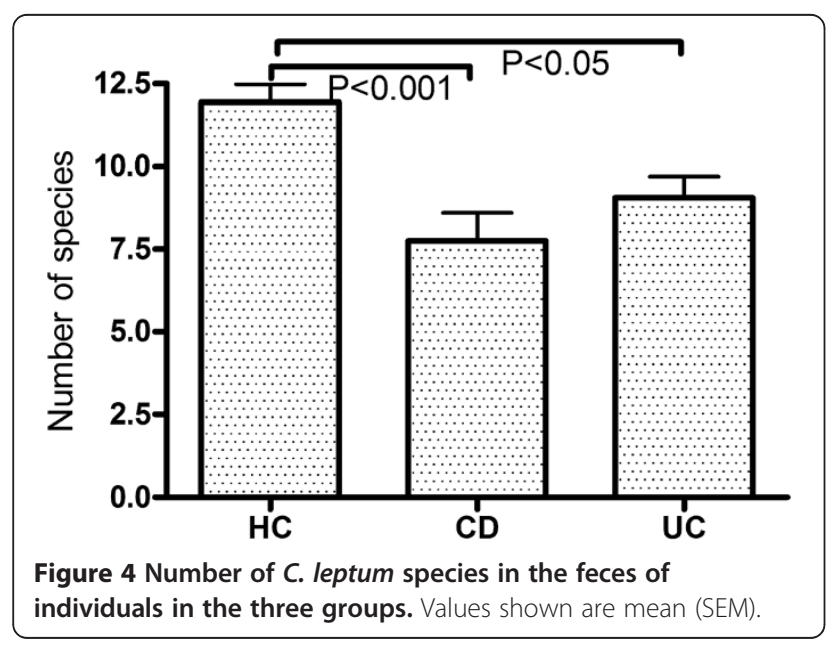

Manichanh et al. [14] reported that the species number of C. leptum, C. coccoides, and certain other Firmicutes was reduced in patients with $\mathrm{CD}$. Their conclusions were based on the analysis of a limited number of clones derived from fecal microbiota of these patients. Other investigators have reported that C. leptum diversity was reduced in patients with $\mathrm{CD}$ [21]. Principal components analysis showed that C. leptum from most of the control subjects clustered discretely to one side whereas C. leptum from CD and UC patients clustered separately. This observation needs to be confirmed in more rigorous studies.

Relative abundance of the targeted microbial communities was evaluated using real time PCR in which the specific abundance was expressed relative to amplification of universal bacterial domain sequences of $16 \mathrm{~S}$ rRNA, i.e. expressed relative to total bacteria. The abundance of 


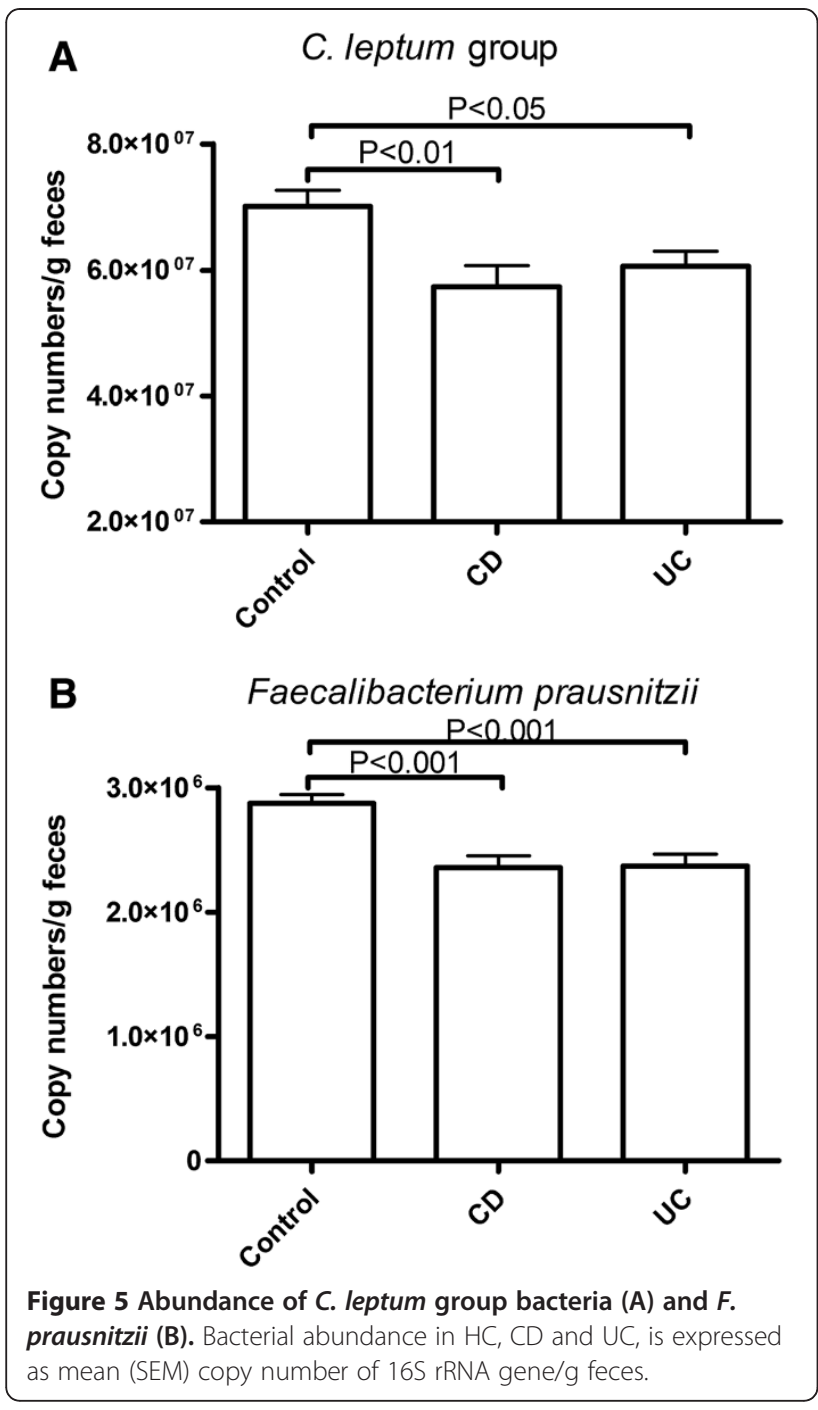

C. leptum group bacteria, and of $F$. prausnitzii, was significantly reduced in both $\mathrm{CD}$ and UC compared to HC. Similar findings have been reported earlier [14,21]. In the present study, there was no significant difference in fecal counts of either C. leptum group or F. prausnitzii between patients with active IBD and those with quiescent IBD (i.e. those who were in remission). There is little literature on this aspect; however one earlier study suggested that the differences in abundance of $C$. leptum and $F$. prausnitzii occurred in active IBD but not in IBD in remission [9].

The significance of these findings can be interpreted varyingly. C. leptum group (cluster IV) is one of the dominant populations of the human fecal microflora $[3,22]$ and contains a large number of butyrate-producing bacteria. It is not known whether the changes in C. leptum number and diversity antedate the development of IBD. However, by showing that changes occur in the same direction in both $\mathrm{CD}$ and $\mathrm{UC}$, we can conclude that these

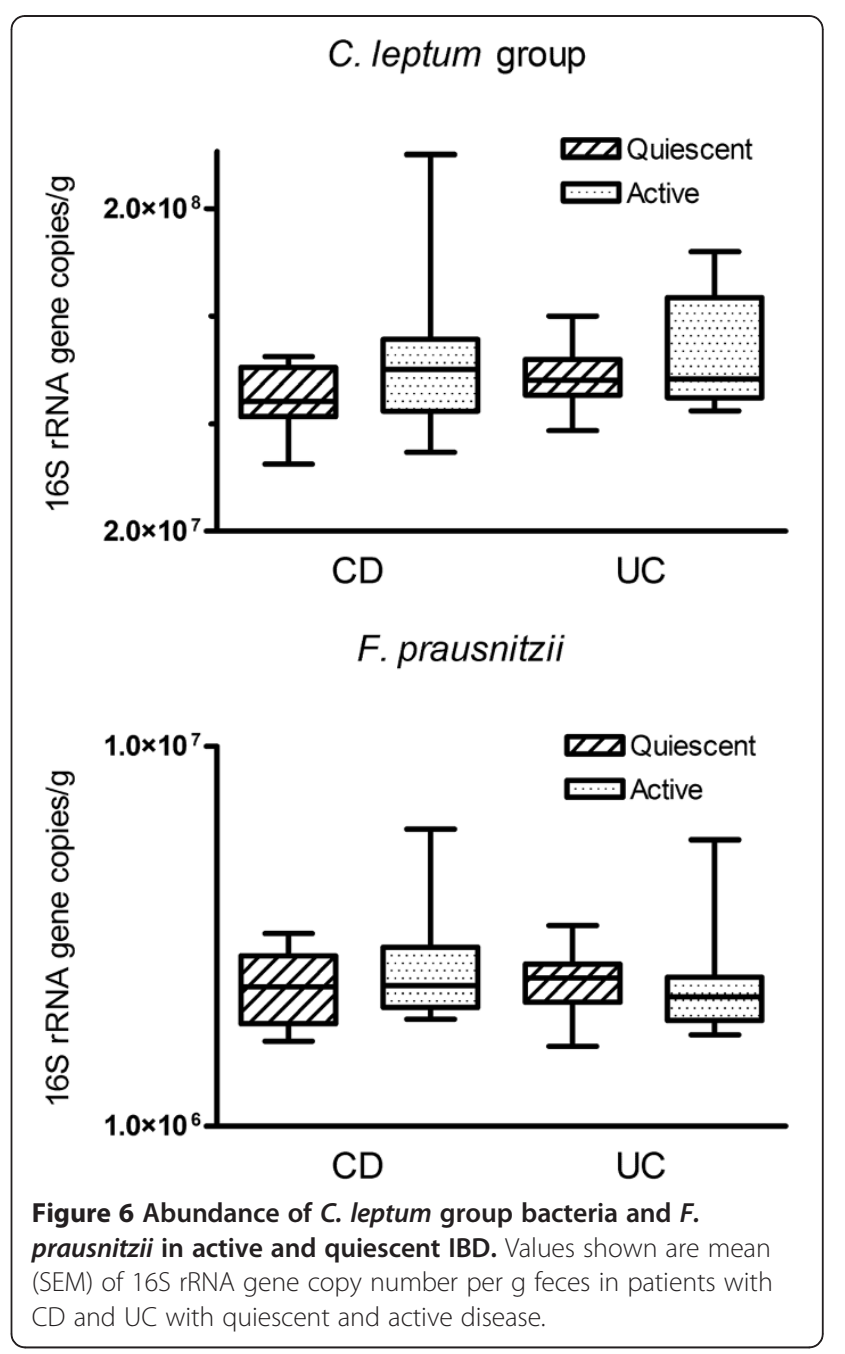

changes are not specific to either disease. The alterations were of greater magnitude in $\mathrm{CD}$ patients compared to UC patients. We do not have an explanation for this observation. It was also noted in our study that the changes were noted in both active and quiescent IBD, suggesting that the changes were not secondary to gut inflammation and ulceration and presence of pus and blood in the lumen. Probiotics used in IBD are typically a mixture of lactobacilli and bifidobacteria. Species belonging to the C. leptum group (esp. F. prausnitzii) are also now considered as anti-inflammatory commensal bacteria. Loss of butyrate, which has an anti-inflammatory activity [23], may result in greater inflammation in the colon.

Our data shows decreased diversity of the C. leptum group in IBD patients. Increased species diversity is an indication of a robust microsystem. Loss of this diversity is likely to impact on gut health, even if it is not the primary phenomenon driving gut inflammation. The intake of prebiotic substrates like inulin can increase both bifidobacteria and $F$. prausnitzii in the human gut, and this may be one possible intervention to consider in 
the management of patients with IBD [24]. It is possible that treatment of IBD patients with butyrate producing bacteria such as the $C$. leptum group bacteria will emerge as another therapeutic option in these patients [25].

\section{Conclusions}

Bacteria belonging to the $C$. leptum group, including the dominant constituent $F$. prausnitzii, are significantly reduced in the fecal microbiota of patients with Crohn's disease and ulcerative colitis. There is a reduction in both the number of species and the absolute number of bacteria in each individual compared to healthy controls. The reduction is found both in active disease and during disease remission, indicating that it is not temporally related to alterations in the intestinal milieu during flares of inflammation. These changes are likely to be significant in the context of the dysbiosis that occurs in patients with IBD.

\section{Abbreviations}

CD: Crohn's disease; HC: Healthy control; IBD: Inflammatory bowel disease; PCR: Polymerase chain reaction; TTGE: Temporal temperature gradient electrophoresis; UC: Ulcerative colitis.

\section{Competing interests}

None of the authors have a financial or non-financial conflict of interest to declare.

\section{Authors' contributions}

JK and BSR contributed to study design, data analysis, and write up of the manuscript. JK, VS and SP contributed to laboratory analyses. BSR obtained financial support for these studies. All authors read and approved the final manuscript.

\section{Acknowledgements}

Dr. Joshua Korzenik and the Washington University School of Medicine provided partial funding support for these studies. JK was a Senior Research Fellow of the Indian Council of Medical Research, New Delhi, India.

Received: 15 August 2012 Accepted: 23 January 2013

Published: 26 January 2013

\section{References}

1. Sghir A, Gramet G, Suau A, Rochet V, Pochart P, Dore J: Quantification of bacterial groups within human fecal flora by oligonucleotide probe hybridization. Appl Environ Microbiol 2000, 66:2263-6.

2. Lay C, Rigottier-Gois L, Holmstrøm K, Rajilic M, Vaughan EE, de Vos WM, Collins MD, Thiel R, Namsolleck P, Blaut M, Doré J: Colonic microbiota signatures across five northern European countries. Appl Environ Microbiol 2005, 71:4153-5.

3. Collins MD, Lawson PA, Willems A, Cordoba JJ, Fernandez-Garayzabal J, Garcia P, Cai J, Hippe H, Farrow JA: The phylogeny of the genus Clostridium: proposal of five new genera and eleven new species combinations. Int J Syst Bacteriol 1994, 44:812-26.

4. Louis P, Flint HJ: Diversity, metabolism and microbial ecology of butyrate-producing bacteria from the human large intestine. FEMS Microbiol Lett 2009, 294:1-8.

5. Sokol H, Pigneur B, Watterlot L, Lakhdari O, Bermúdez-Humarán LG, Gratadoux JJ, Blugeon S, Bridonneau C, Furet JP, Corthier G, Grangette C, Vasquez N, Pochart P, Trugnan G, Thomas G, Blottière HM, Doré J, Marteau $P$, Seksik $P$, Langella P: Faecalibacterium prausnitzii is an antiinflammatory commensal bacterium identified by gut microbiota analysis of Crohn disease patients. Proc Natl Acad Sci USA 2008, 105:16731-6.
6. Sartor RB: Therapeutic manipulation of the enteric microflora in inflammatory bowel diseases: antibiotics, probiotics, and prebiotics. Gastroenterology 2004, 126:1620-33.

7. Cucchiara S, lebba V, Conte MP, Schippa S: The microbiota in inflammatory bowel disease in different age groups. Dig Dis 2009, 27:252-8.

8. Swidsinski A, Loening-Baucke V, Vaneechoutte M, Doerffel Y: Active Crohn's disease and ulcerative colitis can be specifically diagnosed and monitored based on the biostructure of the fecal flora. Inflamm Bowel Dis 2008, 14:147-61.

9. Sokol H, Seksik P, Furet JP, Firmesse O, Nion-Larmurier I, Beaugerie L, Cosnes J, Corthier G, Marteau P, Doré J: Low counts of Faecalibacterium prausnitzii in colitis microbiota. Inflamm Bowel Dis 2009, 15:1183-9.

10. Schwiertz A, Jacobi M, Frick JS, Richter M, Rusch K, Köhler H: Microbiota in pediatric inflammatory bowel disease. J Pediatr 2010, 157:240-244.

11. Mondot S, Kang S, Furet JP, Aguirre de Carcer D, McSweeney C, Morrison M, Marteau P, Doré J, Leclerc M: Highlighting new phylogenetic specificities of Crohn's disease microbiota. Inflamm Bowel Dis 2011, 17:185-92.

12. Kang S, Denman SE, Morrison M, Yu Z, Dore J, Leclerc M, McSweeney CS: Dysbiosis of fecal microbiota in Crohn's disease patients as revealed by a custom phylogenetic microarray. Inflamm Bowel Dis 2010, 16:2034-42.

13. Joossens M, Huys G, Cnockaert M, De Preter V, Verbeke K, Rutgeerts P, Vandamme $P$, Vermeire $S$ : Dysbiosis of the faecal microbiota in patients with Crohn's disease and their unaffected relatives. Gut 2011, 60:631-7.

14. Manichanh C, Rigottier-Gois L, Bonnaud E, Gloux K, Pelletier E, Frangeul L, Nalin R, Jarrin C, Chardon P, Marteau P, Roca J, Dore J: Reduced diversity of faecal microbiota in Crohn's disease revealed by a metagenomic approach. Gut 2006, 55:205-11.

15. Ouyang Q, Tandon R, Goh KL, Pan GZ, Fock KM, Fiocchi C, Lam SK, Xiao SD: Management consensus of inflammatory bowel disease for the AsiaPacific region. J Gastroenterol Hepatol 2006, 21:1772-1782.

16. Best WR, Becktel JM, Singleton JW, Kern F Jr: Development of a Crohn's disease activity index (National Cooperative Crohn's Disease Study). Gastroenterology 1976, 70:439-44.

17. Truelove SC, Witts LJ: Cortisone in ulcerative colitis; final report on a therapeutic trial. Br Med J 1955, 2:1041-8.

18. Shen J, Zhang B, Wei G, Pang X, Wei H, Li M, Zhang Y, Jia W, Zhao L: Molecular profiling of the Clostridium leptum group in human fecal microflora by PCR-denaturing gradient gel electrophoresis and clone library analysis. Appl Environ Microbiol 2006, 72:5232-8.

19. Lay C, Sutren M, Rochet V, Saunier K, Doré J, Rigottier-Gois L: Design and validation of $16 \mathrm{~S}$ rRNA probes to enumerate members of the Clostridium leptum subgroup in human faecal microbiota. Environ Microbiol 2005, 7:933-46.

20. Lay C, Dore J, Rigottier-Gois L: Separation of bacteria of the Clostridium leptum group from the human colonic microbiota by fluorescence-activated cell sorting or group-specific PCR using 16S rRNA gene oligonucleotides. FEMS Microbiol Ecol 2007, 60:513-20.

21. Sokol H, Seksik P, Rigottier-Gois L, Lay C, Lepage P, Podglajen I, Marteau P, Doré J: Specificities of the fecal microbiota in inflammatory bowel disease. Inflamm Bowel Dis 2006, 12:106-11.

22. Matsuki T, Watanabe K, Fujimoto J, Takada T, Tanaka R: Use of $16 \mathrm{~S}$ rRNA gene-targeted group-specific primers for real-time PCR analysis of predominant bacteria in human feces. Appl Environ Microbiol 2004, 70:7220-8.

23. Scheppach W, Weiler F: The butyrate story: old wine in new bottles? Curr Opin Clin Nutr Metab Care 2004, 7:563-7.

24. Ramirez-Farias C, Slezak K, Fuller Z, Duncan A, Holtrop G, Louis P: Effect of inulin on the human gut microbiota: stimulation of Bifidobacterium adolescentis and Faecalibacterium prausnitzii. Br J Nutr 2009, 101:541-50.

25. Van Immerseel F, Ducatelle R, De Vos M, Boon N, Van De Wiele T, Verbeke K, Rutgeerts P, Sas B, Louis P, Flint HJ: Butyric acid-producing anaerobic bacteria as a novel probiotic treatment approach for inflammatory bowel disease. J Med Microbiol 2010, 59:141-3.

doi:10.1186/1471-230X-13-20

Cite this article as: Kabeerdoss et al:: Clostridium leptum group bacteria abundance and diversity in the fecal microbiota of patients with inflammatory bowel disease: a case-control study in India. BMC Gastroenterology 2013 13:20. 